\section{The lateralization of clinical findings might not affect the clinical outcome in multiple sclerosis}

\section{To the Editor}

With great interest, I have read the data published recently by Varoglu and Balkuv. ${ }^{1}$ They raised an interesting clinical question about the outcome and lateralization of clinical findings in patients with Multiple Sclerosis (MS). Several factors need to be considered while looking into the data.

The immunological papers referenced were on animal studies and not on human. ${ }^{2}$ They are investigating the role of cerebral lateralization in the control of immune processes. Similar human studies are few and inconclusive. ${ }^{3}$ The immunological difference in the control by the left and right cerebral hemispheres is a hypothesis. Furthermore, it does not mean a different inflammatory response within the brain.

The main difference between the right and the left cerebral hemispheres relies profoundly on the cognitive ability more than the motor and sensory systems, excluding apraxia and astereognosis; which could be disabling but are rare in MS and not included in the Expanded Disability Status Scale (EDSS) scale., ${ }^{4,5}$ The EDSS, Progression Index (PI), and Multiple Sclerosis Severity Score (MSSS) do not take into account the cognitive symptoms. ${ }^{6,7}$

Some important factors are mentioned in the paper ${ }^{1}$ like age, disease duration, infratentorial lesions, spinal lesions, brainstem lesions, comorbidity, and Progressive Vs. Relapsing MS. These factors were not compared and not statistically adjusted between the left and the right group. For instance, the right clinical finding group might be among older patients with more comorbidities by chance only. Other important factors not mentioned include the use of IV steroids and the type of diseasemodifying treatment.

In Table 3, there are 2 groups of $\mathrm{P}$ values. The horizontal raw compares between the attack and remission. In addition to the vertical column, which compares between the groups (right/left/bilateral). All the $p$-values in the horizontal raw cannot be used to support the conclusion of the paper because it compares between remission and attack. The clinical improvement after the attack is expected due to the decrease in the edema and the inflammatory response after the attack. ${ }^{5}$ Actually, the significant $p$-value in the right clinical finding group supports a better recovery and not a worse outcome. To illustrate, the significant $p$-value in Table 3 (0.008) showed that the attack is worse than the remission, which means better recovery. The $p$-value was significant for the right side but not on the left side. This contradicts the conclusion; the clinical improvement is better in patients with the right clinical findings. The $p$-value in the vertical column reflects the statistical difference between the groups (right/ left/bilateral). No significant $p$-value related to the EDSS, but there are statistically significant differences in PI and MSSS. The PI and MSSS cannot be used to support the conclusion for multiple reasons. First, the PI and MSSS are calculated from the EDSS and taking into account the time factor. ${ }^{6,7}$ So, we cannot make a fair comparison without adjusting for disease duration between the right and the left group. Second, if they showed statistical significance, one should expect the same for EDSS. Third, it was only significant at the first attack, not the last one, which is partially expected as these measures are affected by the time and the natural history of the disease. ${ }^{5}$

The MS is a multifocal disease, and hence most of the patients (259) had bilateral symptoms at the last attack while only 15 patients had lateralized findings. This makes it difficult to make a conclusion based on a very small number of cases. On the other hand, It is hard to consider the group with bilateral clinical findings as "not associated with the poor outcome" because the majority of the patients in this cohort and real practice will have bilateral involvement due to the multifocal nature of the disease. ${ }^{5}$ The MSSS score is defined from 0 to $10,{ }^{7}$ but the reported numbers in Tables $\mathbf{2} \& \mathbf{3}$ are exceeding the maximum score. There is no precise time definition of the remission period. A definition of 30 days is quite different than 90 days in terms of recovery. Moreover, this information is very difficult to obtain in a retrospective study design.

The best study to answer this intriguing question is to consider all the confounders mentioned above, and to use other scales that focus not only on the motor symptoms, but also on the cognitive ones. Above all, these factors need to be measured prospectively. But until then, the right side clinical finding is not necessarily associated with a worse clinical outcome in MS.

Abmad A. Abulaban, Department of Medicine, Neurology Division, King Saud BinAbdulaziz University for Health Sciences, National Guard Health Affairs, Riyadh, Kingdom of SaudiArabia

\section{Reply from the Author}

No reply received from the author 


\section{References}

1. Varoglu AO, Balkuv E. Right-Sided clinical findings are worse prognostic factor in Multiple Sclerosis patients? Neurosciences (Riyadh) 2020; 25: 97-103.

2. Gontova IA, Abramov VV, Kozlov VA. The role of asymmetry of nervous and immune systems in the formation of cellular immunity of (CBaxC57Bl/6) F1 mice. Neuroimmunomodulation 2004; 11: 385-391.

3. Meador KJ, Loring DW, Ray PG, Helman SW, Vazquez BR, Neveu PJ. Role of cerebral lateralization in control of immune processes in humans. Ann Neurol 2004; 55: 840-844.
4. Blumenfeld Hal. Neuroanatomy Through Clinical Cases. 2nd edition. Sunderland (MA): Sinauer Associates Inc; 2010.

5. Files DK, Jausurawong T, Katrajian R, Danoff R. Multiple sclerosis. Prim Care 2015; 42: 159-75.

6. Kurtzke JF. Rating neurologic impairment in multiple sclerosis: an Expanded Disability Status Scale (EDSS). Neurology 1983; 33: 1444-1452.

7. Roxburgh RH, Seaman SR, Masterman T, Hensiek AE, Sawcer SJ, Vukusic S. Multiple Sclerosis Severity Score: using disability and disease duration to rate disease severity. Neurology 2005; 64: 1144-1151.

\section{Withdrawal policy}

By submission, the author grants the journal right of first publication. Therefore, the journal discourages unethical withdrawal of manuscripts from the publication process after peer review. The corresponding author should send a formal request signed by all co-authors stating the reason for withdrawing the manuscript. Withdrawal of a manuscript is only considered valid when the editor accepts, or approves the reason to withdraw the manuscript from publication. Subsequently, the author must receive a confirmation from the editorial office. Only at that stage, are the authors free to submit the manuscript elsewhere.

No response from the authors to all journal communication after review and acceptance is also considered unethical withdrawal. Withdrawn manuscripts noted to have already been submitted or published in another journal will be subjected to sanctions in accordance with the journal policy. The journal will take disciplinary measures for unacceptable withdrawal of manuscripts. An embargo of 5 years will be enforced for the author and their co-authors, and their institute will be notified of this action. 